\title{
Physical impairments modeling for the performance evaluation of transparent optical networks
}

\author{
Helder A. Pereira, Daniel A. R. Chaves, Carmelo J. A. Bastos-Filho and Joaquim F. Martins-Filho
}

\begin{abstract}
We propose a model to consider several physical impairments in optical networks based on the OSNR. Our model considers the effects of gain saturation and amplified spontaneous emission noise in amplifiers, homodyne crosstalk in optical switches, and four wave mixing in the transmission fibers. We present an application of our model for the evaluation of network performance in terms of blocking probability using a routing and wavelength assignment algorithm. Our results show the impact of each impairment on network performance as a function of device parameters.
\end{abstract}

Keywords-All-Optical Networks, Noise, Optical Signal-toNoise Ratio, Routing and Wavelength Assignment.

\section{INTRODUCTION}

All-optical networks have been considered as the most reliable and economic solution to achieve high transmission capacities. In these networks, the signal remains in the optical domain between the edge nodes, i.e., the signal propagates along the optical network without suffering any opticalelectrical-optical conversion.

In a circuit switched scenario, each wavelength is treated as a single call that can be routed through multiple links in the network to reach the destination node. Therefore, it is necessary to implement routing processes in the network, which is accomplished by the optical cross-connectors (OXC). The OXC are connected to the network control plane and depending on the routing process it can improve the network performance in terms of blocking probability of requested calls $[1 ; 2]$.

There are two main challenges to manage these networks providing quality of service (QoS): design an appropriate routing and wavelength assignment algorithm (RWA) and provide an acceptable optical signal-to-noise ratio (OSNR) for every optical signal. The physical impairments can lead to optical OSNR degradation, which have impact on the QoS [35].

The main physical impairments that impact the optical network performance are the amplifier saturation and amplified spontaneous emission (ASE) in optical amplifiers, homodyne crosstalk in switches, chromatic dispersion, polarization mode dispersion (PMD) and nonlinear effects in fibers [2; 6-12].

In this paper we propose a model based on the OSNR degradation to consider several physical impairments in optical

Helder A. Pereira is with the Department of Electrical Engineering, University of Pernambuco, 52720-001, Recife-PE, Brazil. Daniel A. R. Chaves and Joaquim F. Martins-Filho are with Photonic's Group, Department of Electronics and Systems, Federal University of Pernambuco, 50740-530, Recife-PE, Brazil. E-mail: jfmf@ufpe.br. Carmelo J. A. Bastos-Filho is with the Department of Computing Systems, University of Pernambuco, 52720001, Recife-PE, Brazil. networks. We present an application of our model for the evaluation of network performance in terms of blocking probability using a well known routing and wavelength assignment algorithm. Our results show the impact of each impairment on network performance as a function of device parameters. In Section II, we describe our model that considers physical impairments in all-optical networks. In Section III, we present general characteristics and parameters used in our simulations considering a specific optical network topology. In Section IV we show the simulation results. In Section V we give our conclusions.

\section{PHYSICAL IMPAIRMENTS MODELING}

Our formulation quantifies the OSNR degradation along the optical signal propagation in the all-optical network. The impact of physical layer impairments is taken into account by considering the signal power and the noise power at the destination node, both affected by gains and losses along the lightpath. Moreover, network elements add noise components. The optical amplifiers add ASE noise power and also suffer from gain saturation and ASE depletion as the total signal power increases. The optical switches add noise due to nonideal isolation between ports. And the transmission fibers add noise due to four wave mixing (FWM) and also induces pulse broadening due to PMD. We neglect the effect of chromatic dispersion since we assume that GVD is totally compensated in the network links.

Fig. 1 shows the network devices considered in our model in each link. The links has the following elements: transmitter, optical switch, multiplexer, booster amplifier, optical fiber, pre-amplifier, demultiplexer, optical switch and receiver. The points $a$ until $h$ are measurement points where the signal and noise can be determined in the optical domain. In point $a$, we have the input optical signal power $\left(P_{i n}\right)$ and the input optical noise power $\left(N_{i n}\right)$. The ratio between $P_{i n}$ and $N_{i n}$ defines the OSNR of the transmitter $\left(\mathrm{OSNR}_{i n}\right)$. For the lightpath with $k$ links, the elements between $b$ and $h$ are repeated $k$ times before the signal reaches the receiver in the destination node.

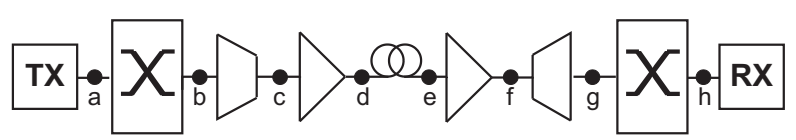

Fig. 1. The link configuration with optical devices considered in our model.

At points $b$ and $h$, we consider the noise induced by homodyne crosstalk in the optical switch. This occurs basically because the energy of one optical signal can leak to other 
co-propagating signals due to non-ideal optical switches. The optical noise power generated by each optical switch in every wavelength is given by [1]

$$
N_{\text {Switch }}=\varepsilon \sum_{j=1}^{n} P_{S w_{j}}(\lambda),
$$

where $P_{S w_{j}}(\lambda)$ is the received optical power from the $\mathrm{j} t h$ optical fiber in the same wavelength of the propagating optical signal, $\varepsilon$ is the switch isolation factor and $n$ is the number of signals in the same wavelength received from others links. At points $c$ and $g$, we just considered the multiplexer and demultiplexer losses.

At points $d$ and $f$, we take into account the noise induced by the optical amplifiers, as well as the gain saturation effect. Considering the signal-spontaneous beating as the main noise source, this noise can be quantified by [13]

$$
N_{a m p}=\frac{h \nu(\lambda) B_{o} G_{a m p} F_{a m p}}{2},
$$

where $h$ is the Planck constant, $\nu(\lambda)$ is the optical signal frequency, $B_{o}$ is the optical filter bandwidth, $G_{a m p}$ is the dynamic amplifier gain and $F_{a m p}$ is the amplifier noise factor.

The gain saturation effect is taking into account by using the following expression $[4 ; 5]$

$$
G_{a m p}=\frac{G_{0}}{1+\frac{P_{\text {out }}}{P_{\text {sat }}}},
$$

where $G_{0}$ is the maximum non-saturated amplifier gain, $P_{\text {out }}$ is the optical power at the amplifier output and $P_{\text {sat }}$ is the amplifier output saturation power.

Since $F_{a m p}$ depends on the input optical power, we developed the following expression:

$$
F_{a m p}=F_{0}\left(1+A_{1}-\frac{A_{1}}{1+\frac{P_{i n}}{A_{2}}}\right),
$$

where $F_{0}$ is the amplifier noise factor for low input optical powers, $A_{1}$ and $A_{2}$ are function parameters. These parameters were obtained by fitting experimental results from an erbium doped fiber amplifier (EDFA) developed in our laboratories.

Fig. 2 shows the amplifier gain and amplifier noise figure as a function of input optical power per channel. The experimental results are represented by symbols and the model results are represented by solid curves. The function parameters that fit the experimental results of Fig. 2 are: $G_{0}^{d B}=30 \mathrm{~dB}, F_{0}=3(N F=4.77 \mathrm{~dB}), P_{\text {sat }}=15 \mathrm{dBm}$, $A_{1}=500$ and $A_{2}=2 \mathrm{~W}$.

In real optical amplifiers, both the ASE and the amplifier gain diminish as the input optical power increases. However, the amplifier gain decays faster than the ASE. As a consequence, using Eq. (4), one can observe that the noise figure increases with the input optical power. To our knowledge, we are the first to consider the dependence of gain, noise factor and amplifier noise power with the signal power for all-optical networks performance evaluation.

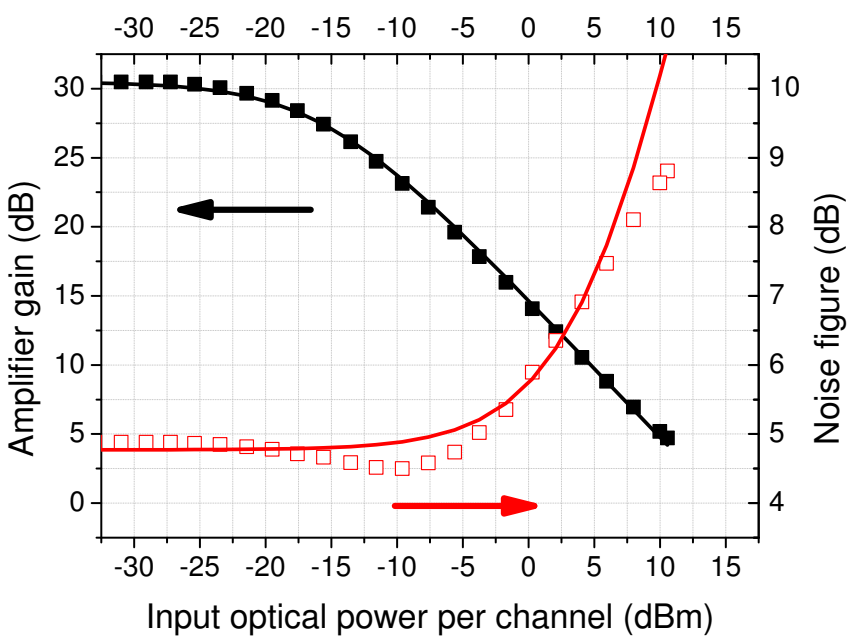

Fig. 2. Amplifier gain and amplifier noise figure as a function of input optical power per channel obtained from experimental results and fitting model.

At point $e$, we consider the noise generated by FWM effect [2]. This nonlinear effect depends on the channel spacing, optical signal power per channel, number of wavelengths propagating in optical fiber, fiber dispersion coefficient, phase matching and the zero dispersion wavelength of the fiber. The FWM generated power can be evaluated using the formulation proposed by Song et al [14], using equations (1-5) therein. Considering every optical power component generated by FWM in the respective signal wavelength, we have

$$
N_{F W M}=\sum_{j=1}^{m} P_{F W M_{j}}(\lambda),
$$

where $N_{F W M}$ is the noise power due to FWM, $P_{F W M_{j}}(\lambda)$ is one of the $m$ optical power component generated by FWM effect that falls into the same propagating signal wavelength.

Finally, at the point $h$, one can evaluate the output optical signal power $\left(P_{\text {out }}\right)$ and the output optical noise power $\left(N_{\text {out }}\right)$. $P_{\text {out }}$ is evaluated according to the gains and losses along the signal propagation and it is given by

$$
P_{\text {out }}=\frac{G_{a m p_{1}} e^{-\alpha d} G_{a m p_{2}}}{L_{\text {Switch }}^{2} L_{M u x} L_{\text {Demux }}} P_{i n}
$$

where $G_{a m p_{1}}$ and $G_{a m p_{2}}$ are the dynamic linear gains of the booster and pre-amplifier, $\alpha$ is the fiber loss coefficient, $d$ is the fiber length, $L_{\text {Switch }}, L_{M u x}$ and $L_{\text {Demux }}$ are the optical switch, multiplexer and demultiplexer losses.

$N_{\text {out }}$ is evaluated from the source node to the destination node, including the additive noise component in the respective points along the lightpath and is given by 


$$
\begin{aligned}
N_{\text {out }}= & \frac{G_{a m p_{1}} e^{-\alpha d} G_{a m p_{2}}}{L_{M u x} L_{\text {Demux }} L_{\text {Switch }}^{2}} N_{\text {in }}+ \\
& +\frac{G_{a m p_{1}} e^{-\alpha d} G_{a m p_{2}}}{L_{M u x} L_{\text {Demux }} L_{\text {Switch }}} \varepsilon \sum_{j=1}^{n} P_{S w_{1, j}}(\lambda)+ \\
& +\frac{G_{a m p_{1}} e^{-\alpha d} G_{a m p_{2}}}{L_{\text {Demux }} L_{\text {Switch }}} \\
& \frac{h \nu(\lambda) B_{o}}{2}\left(F_{a m p_{1}}+\frac{F_{a m p_{2}}}{e^{-\alpha d} G_{a m p_{1}}}\right)+ \\
& +\frac{G_{a m p_{2}}}{L_{D e m u x} L_{S w i t c h}} \sum_{j=1}^{m} P_{F W M_{j}}(\lambda)+ \\
& +\varepsilon \sum_{j=1}^{s} P_{S w_{2, j}}(\lambda)
\end{aligned}
$$

where $N_{i n}$ is the noise power at the transmitter output.

Dividing $P_{\text {out }}$ by $N_{\text {out }}$, one can obtain the OSNR at destination node $\left(\mathrm{OSNR}_{\text {out }}\right)$. The $\mathrm{OSNR}_{\text {out }}$ is related directly to the BER [15]. Therefore, one can establish a threshold OSNR that guarantees the QoS $\left(\mathrm{OSNR}_{Q o S}\right)$ for call requests on the network.

Considering a route with a number of $i$ links, we have

$$
P_{\text {out }_{i}}=\left(\frac{G_{a m p_{i, 1}} e^{-\alpha d_{i}} G_{a m p_{i, 2}}}{L_{M u x} L_{\text {Demux }} L_{\text {Switch }}}\right) P_{\text {out }_{i-1}}
$$

and

$$
\begin{aligned}
N_{\text {out }_{i}}= & \frac{G_{a m p_{1, i}} e^{-\alpha d_{i}} G_{a m p_{2, i}}}{L_{M u x} L_{\text {Demux }} L_{\text {Switch }}} N_{\text {out }_{i-1}}+ \\
& +\frac{G_{a m p_{1, i}} e^{-\alpha d_{i}} G_{a m p_{2, i}}}{L_{\text {Demux }} L_{\text {Switch }}} \frac{h \nu(\lambda) B_{o}}{2} \\
& \left(F_{a m p_{1, i}}+\frac{F_{a m p_{2, i}}}{e^{-\alpha d_{i}} G_{a m p_{1, i}}}\right)+ \\
& +\frac{G_{a m p_{2, i}}}{L_{\text {Demux }} L_{S w i t c h}} \sum_{j=1}^{m} P_{F W M_{i, j}}(\lambda)+ \\
& +\varepsilon \sum_{j=1}^{s} P_{S w_{i+1, j}}(\lambda)
\end{aligned}
$$

$$
\text { where } N_{\text {out }_{0}}=\frac{N_{\text {in }}}{L_{\text {Switch }}}+\varepsilon \sum_{j=1}^{n} P_{S w_{1, j}}(\lambda) \text { and }
$$$$
P_{\text {out }_{0}}=\frac{P_{\text {in }}}{L_{\text {Switch }}} \text {. }
$$

Furthermore, we consider the pulse broadening caused by PMD effect in a route using the following expression [16],

$$
\Delta t=B \sqrt{\sum_{j=1}^{i} D_{P M D}(j) d(j)}
$$

where $B$ is the transmission bit rate, $D_{P M D}(j)$ is the fiber PMD coefficient, and $d(j)$ is the fiber length in the jth link belonging to the lightpath. The $\Delta t$ should be lower than a pre-determined maximum pulse broadening $(\delta)$.

\section{Simulation CHARACTERISTICS}

Fig. 3 shows the flowchart of our simulation algorithm. For each network simulation, a set of $10^{5}$ calls are generated choosing randomly the source-destination pair. The call request process is characterized as a Poisson process. For each call request the first available wavelength is assigned between the source and destination nodes (first-fit algorithm) and the route is determined using shortest path algorithm [3].

Our algorithm blocks a call if there is no wavelength available, if the OSNR for the respective wavelength is below of $\operatorname{OSNR}_{Q o S}$, or if the pulse broadening $(\Delta t)$ is above the maximum level $(\delta)$. The blocked calls are lost. The blocking probability is obtained from the ratio of the number of blocked calls and the number of call requests. We assume circuit-switched bidirectional connections in two fibers and no wavelength conversion capabilities. The default parameters used in our simulations are shown in Tabel I and Fig. 4 shows the analyzed optical network topology. In each link the amplifier gains are set to compensate for the total link losses.

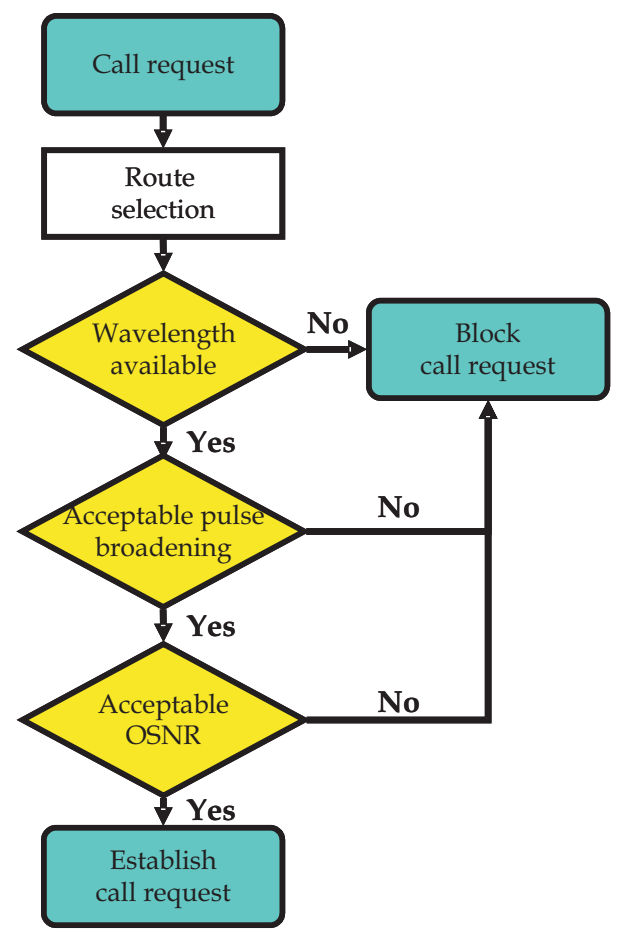

Fig. 3. Flow chart of the routing and wavelength assignment algorithm employed in our network simulations.

\section{RESUlts}

We present simulation results for network blocking probability as a function of input optical power per channel, for different network device characteristics.

Fig. 5 shows the blocking probability as a function of input optical power per channel for different amplifier noise figure values. When amplifiers with $N F=3 \mathrm{~dB}$ are used, we have lower blocking probability and we obtain the minimum blocking probability with an input optical power per channel of around $-2 \mathrm{dBm}$. For laser transmitters with optical power higher than $-2 \mathrm{dBm}$ we verified that homodyne crosstalk, 
TABLE I

SiMULATION PARAMETERS.

\begin{tabular}{|c|c|c|}
\hline Parameter & Value & Definition \\
\hline$\overline{\bar{P} P_{\text {Sat }}}$ & $16 \mathrm{dBm}$ & $\begin{array}{l}\text { Amplifier output } \\
\text { saturation power. }\end{array}$ \\
\hline $\mathrm{OSNR}_{\text {in }}$ & $30 \mathrm{~dB}$ & $\begin{array}{l}\text { Input optical } \\
\text { signal-to-noise ratio. }\end{array}$ \\
\hline $\mathrm{OSNR}_{Q o S}$ & $23 \mathrm{~dB}$ & $\begin{array}{l}\text { Optical signal-to-noise } \\
\text { ratio for QoS } \\
\text { criterion. }\end{array}$ \\
\hline$B$ & $40 \mathrm{Gbps}$ & Transmission bit rate. \\
\hline$\overline{B_{o}}$ & $100 \mathrm{GHz}$ & $\begin{array}{l}\text { Optical filter } \\
\text { bandwidth. }\end{array}$ \\
\hline$W$ & 36 & $\begin{array}{l}\text { Number of wavelengths } \\
\text { in an optical link. }\end{array}$ \\
\hline$\Delta f$ & $100 \mathrm{GHz}$ & Channel spacing. \\
\hline$\lambda_{i}$ & $1550.12 \mathrm{~nm}$ & $\begin{array}{l}\text { The lower wavelength of } \\
\text { the grid. }\end{array}$ \\
\hline$\lambda_{0}$ & $1510 \mathrm{~nm}$ & $\begin{array}{l}\text { Zero dispersion } \\
\text { wavelength. }\end{array}$ \\
\hline$\alpha$ & $0.2 \mathrm{~dB} / \mathrm{km}$ & Fiber loss coefficient. \\
\hline$L_{M u x}$ & $3 \mathrm{~dB}$ & Multiplexer loss. \\
\hline$\overline{L_{\text {Demux }}}$ & $3 \mathrm{~dB}$ & Demultiplexer loss. \\
\hline$L_{\text {Switch }}$ & $3 \mathrm{~dB}$ & Switch loss. \\
\hline$F_{0}$ & 3.162 & $\begin{array}{l}\text { Amplifier noise factor } \\
\text { that corresponds to } \\
N F=5 \mathrm{~dB} .\end{array}$ \\
\hline$\overline{A_{1}}$ & 100 & $\begin{array}{l}\text { Noise factor model } \\
\text { parameter. }\end{array}$ \\
\hline$A_{2}$ & $4 \mathrm{~W}$ & $\begin{array}{l}\text { Noise factor model } \\
\text { parameter. }\end{array}$ \\
\hline$\epsilon$ & $-40 \mathrm{~dB}$ & $\begin{array}{l}\text { Switch isolation } \\
\text { factor. }\end{array}$ \\
\hline$\delta$ & $10 \%$ & $\begin{array}{l}\text { Maximum pulse } \\
\text { broadening. }\end{array}$ \\
\hline$D_{P M D}$ & $0.05 \mathrm{ps} / \mathrm{km}^{1 / 2}$ & $\begin{array}{l}\text { PMD dispersion } \\
\text { coefficient. }\end{array}$ \\
\hline Load & 60 Erlangs & Network load. \\
\hline
\end{tabular}

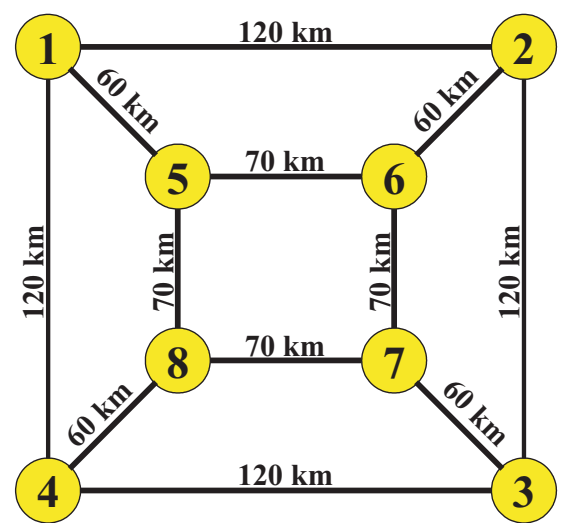

Fig. 4. The optical network used in our simulations. Node distances are shown.

amplifier saturation gain and FWM effect cause more impact on OSNR degradation. For laser transmitters with optical power lower than $-2 \mathrm{dBm}$, the blocking probability increases because of the ASE impact. For $N F=5 \mathrm{~dB}$, the ideal input optical power per channel value is $-1 \mathrm{dBm}$ and for $N F=7 \mathrm{~dB}$ it is $0 \mathrm{dBm}$. Note the dependence of the optimum input optical power per channel with the amplifiers noise figure.

Fig. 6 shows the blocking probability as a function of input optical power per channel for different amplifier output

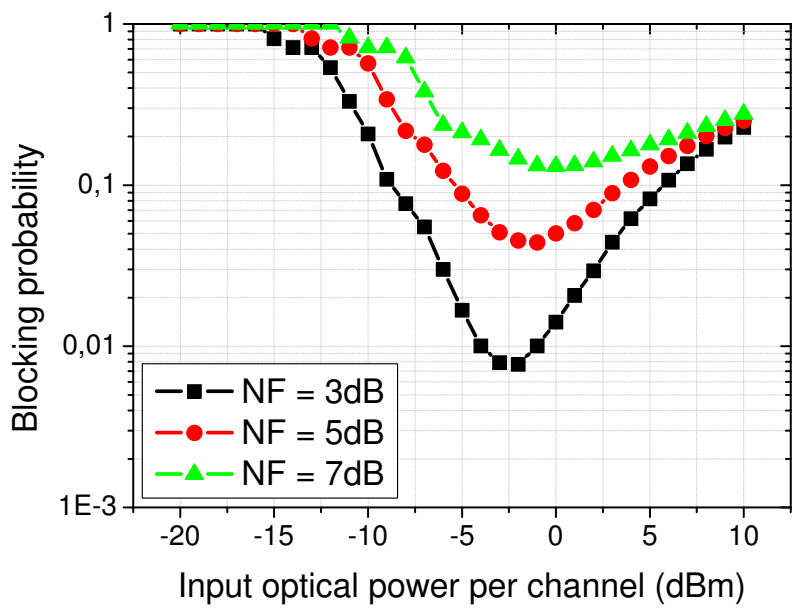

Fig. 5. Blocking probability as a function of input optical power per channel for different amplifier noise figure values.

saturation power values. The input optical power per channel that corresponds to minimum blocking probability is equal to $0 \mathrm{dBm}$. For a amplifier that has $P_{\text {out }}=13 \mathrm{dBm}$, we have $16.81 \%$ of blocked calls, for $P_{\text {out }}=16 \mathrm{dBm}$, we have $4.41 \%$ and for $P_{\text {out }}=19 \mathrm{dBm}$, we have around $0.33 \%$ of call blocking. Note that a $3 \mathrm{~dB}$ increase in the amplifier saturation power leads to a reduction in the blocking probability by more than 1 order of magnitude.

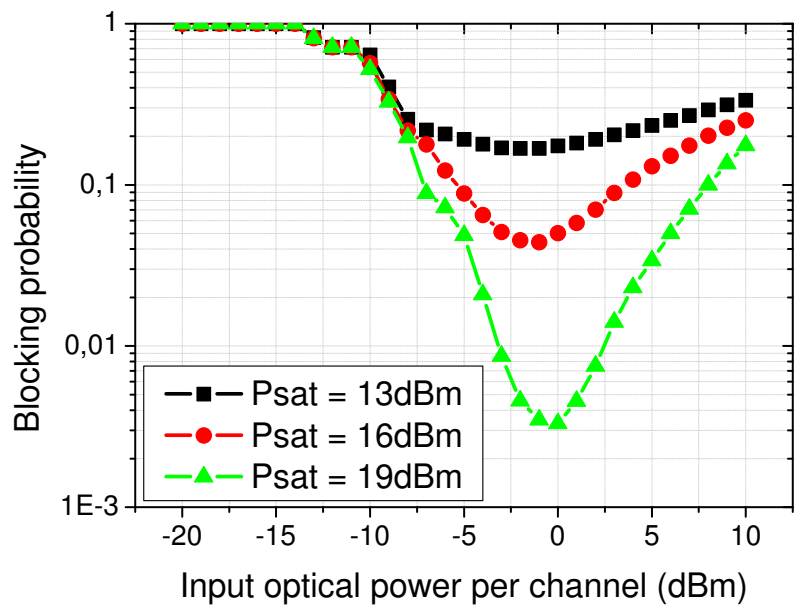

Fig. 6. Blocking probability as a function of input optical power per channel for different amplifier output saturation power values.

There are different types of fiber installed in the optical communication systems all over the world. To analyze the network performance with different types of fiber, we considered the standard fiber (STD), with $\lambda_{0}=1310 \mathrm{~nm}$, dispersion shifted fiber (DSF), with $\lambda_{0}=1550 \mathrm{~nm}$, and nonzero dispersion shifted fiber (NZ-DSF), with $\lambda_{0}=1510 \mathrm{~nm}$. Fig. 7 shows the blocking probability as a function of input optical power per channel for different zero dispersion wavelength values. For any fiber type the blocking probability increases as the signal power increases due to FWM. We can note that there is no difference in network performance when using NZ-DSF or STD fibers, for a transmission bit rate of $40 \mathrm{Gbps}$ and channel spacing of $100 \mathrm{GHz}$. However, 
when DSF fiber is considered, we have more blocked calls caused by FWM effect. For laser transmitters with optical powers lower than $-9 \mathrm{dBm}$ we note that the type of the optical fiber employed in the network does not affect the network performance, since the calls are blocked mainly due to accumulation of ASE noise.

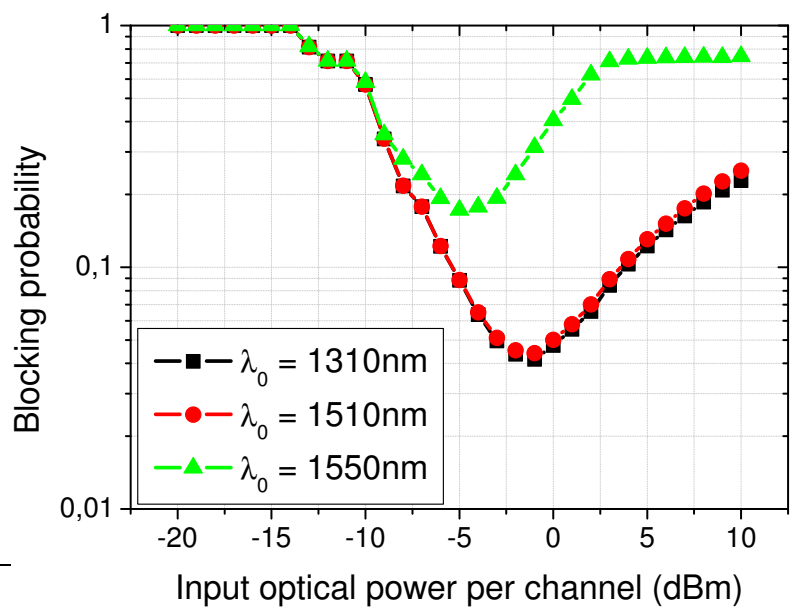

Fig. 7. Blocking probability as a function of input optical power per channel for different zero dispersion wavelength values.

Fig. 8 shows the blocking probability as a function of input optical power per channel for different switch isolation factors. The network performance is very sensitive to this parameter. For $\varepsilon=-40 \mathrm{~dB}$ we have $4.41 \%$ of blocked calls, while for $\varepsilon=-35 \mathrm{~dB}$ we have $10.15 \%$. Considering $\varepsilon=-30 \mathrm{~dB}$ we have more than $45.94 \%$ blocked calls for the input optical power per channel equal to $-1 \mathrm{dBm}$.

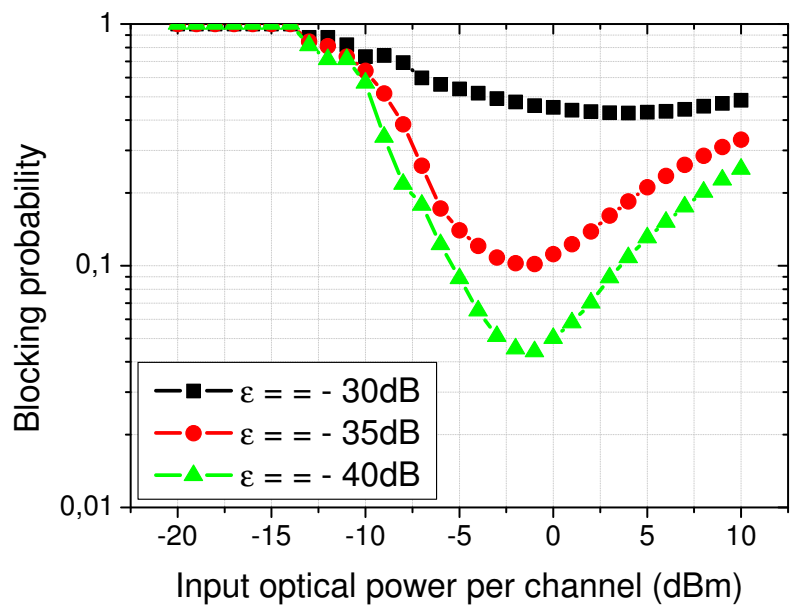

Fig. 8. Blocking probability as a function of input optical power per channel for different switch isolation factors.

Fig. 9 shows the blocking probability as a function of input optical power per channel for different network loads. As expected, the blocking probability increases as the network traffic load increases. However, Fig. 9 shows that the minimum blocking probability for each load occurs for different values of input optical signal powers. This is because of the balance between the different effects. For high loads more wavelengths are used. Therefore, the FWM effect and the switch noise increase, which causes the shift of the minimum blocking probability towards lower input optical powers.

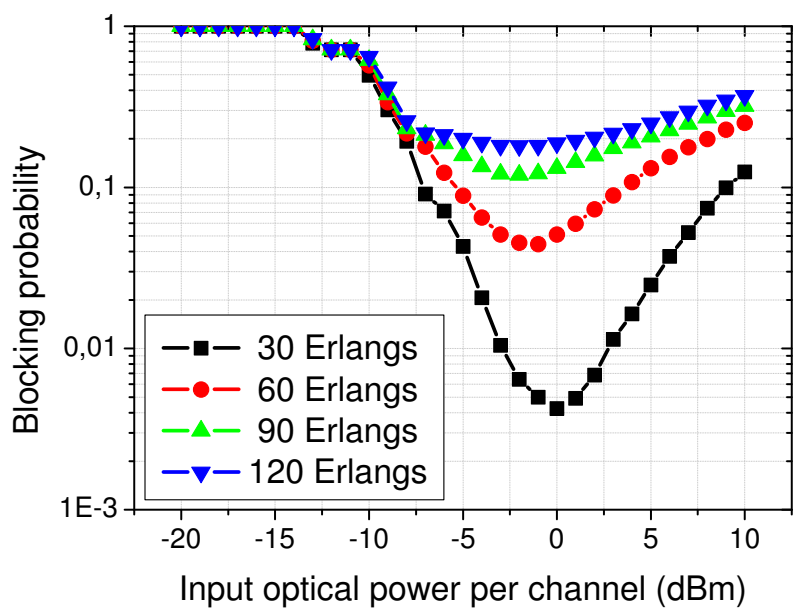

Fig. 9. Blocking probability as a function of input optical power per channel for different network loads.

\section{CONCLUSiOnS}

We presented a novel model to consider several physical impairments in transparent optical networks. Our model is based on the degradation of OSNR along the lightpaths and it considers the effects of gain saturation and amplified spontaneous emission noise in amplifiers, homodyne crosstalk in optical switches, and four wave mixing in the transmission fibers.

To our knowledge, we are the first to propose a simple model based on OSNR to consider these effects all together, using analytical equations obtained from well known fundamental or experimental behavior of network devices. Moreover, we are also the first to consider de dependence of gain, noise factor and overall amplifier noise power on the total signal power. We presented an application of our model for the evaluation of network performance in terms of blocking probability using a routing (shortest path) and wavelength assignment (first fit) algorithms.

Our results show the impact of each impairment on network performance as a function of device parameters. For low signal powers the blocking probability is mainly due to the amplifiers noise, whereas for high signal powers the main contribution to the blocking probability comes from the FWM effect. We note that the optimum signal power depends on network topology and network device parameters.

Our simulation results also show that network performance is highly dependent on device parameters, such as amplifier output saturation power, amplifier noise figure, switch isolation factor, and fiber type. These device parameters have considerable impact on device costs. Therefore, we believe our model have application in routing and wavelength assignment algorithms, and also in network planning to balance costs and performance.

\section{ACKNOWLEDGMENTS}

The authors acknowledge the financial support from $\mathrm{CNPq}$ and Capes (Brazilian Research Council). 


\section{REFERÊNCIAS}

[1] R. Ramaswami and K. N. Sivarajan, Optical Networks: A Practical Perspective, 2nd ed. Morgan Kaufmann, 2002.

[2] G. P. Agrawal, Fiber-Optic Communication Systems, 2nd ed. John Wiley and Sons, Inc., 1997.

[3] H. Zang, J. P. Jue, and B. Mukherjee, "A review of routing and Wavelength assignment approaches for wavelength-routed optical wdm networks," Optical Networks Magazine - SPIE/Kluwer Publishers, vol. 1, no. 1, January 1999.

[4] J. F. Martins-Filho, C. J. A. Bastos-Filho, E. A. J. Arantes, S. C. Oliveira, L. D. Coelho, J. P. G. de Oliveira, R. G. Dante, E. Fontana, and F. D. Nunes, "Novel routing algorithm for transparent optical networks based on noise figure and amplifier saturation," Proceedings of IMOC, vol. 2, pp. 919-923, September 2003.

[5] J. F. Martins-Filho, C. J. A. Bastos-Filho, S. C. Oliveira, E. A. J. Arantes, E. Fontana, and F. D. Nunes, "Novel routing algorithm for optical networks based on noise figure and physical impairments," in Proceedings of ECOC, vol. 3. OSA, 2003, pp. 856-857.

[6] B. Ramamurthy, D. Datta, H. Feng, J. P. Heritage, and B. Mukherjee, "Impact of transmission impairments on the teletraffic performance of wavelength-routed optical networks," Journal of Lightwave Technology, vol. 17, no. 10, pp. 1713-1723, October 1999.

[7] E. Karasan and E. Ayanoglu, "Effects of Wavelength Routing and selection algorithms on wavelength Conversion gain in wdm optical networks," IEEE/ACM Transactions on Networking, vol. 6, no. 2, pp. 186-196, April 1998.

[8] I. Tomkos, D. Vogiatzis, C. Mas, I. Zacharopoulos, A. Tzanakaki, and E. Varvarigos, "Performance engineering of metropolitan area optical networks through impairment constraint routing," IEEE Communications Magazine, vol. 42, no. 8, pp. S40-S47, August 2004.

[9] M. Ali, B. Ramamurthy, and J. S. Deogun, "Routing and wavelength assignment with power considerations in optical networks," Elsevier Computer Networks, vol. 32, no. 5, pp. 539-555, May 2000.

[10] I. E. Fonseca, M. R. N. Ribeiro, R. C. Almeida Jr., and H. Waldman, "Preserving global optical qos in fwm impaired dynamic networks," Eletronics Letters, vol. 40, no. 3, pp. 191-192, February 2004.

[11] M. Ali and L. Tancevski, "Impact of polarization-mode dispersion on the design of wavelength-routed networks," Photonics Technology Letters, vol. 14, pp. 720-722, May 2002.

[12] M. A. et al, "Network optimization with transmission impairments-based routing," in Proceedings of ECOC. OSA, 2001, pp. 42-43.

[13] D. M. Baney, P. Gallion, and R. S. Tucker, "Theory and measurement techniques for the noise figure of optical amplifiers," Optical Fiber Technology, vol. 6, pp. 122154, 2000.
[14] S. Song, C. Allen, K. Demarest, and R. Hui, "Intensitydependent phase-matching effects on four-wave mixing in optical fibers," Journal of Lightwave Technology, vol. 17, no. 11, pp. 2285-2290, November 1999.

[15] K. Thyagarajan and A. K. Ghatak, An Introduction to Fiber Optics, 1st ed. Cambridge - USA, 1998.

[16] J. Strand, A. L. Chiu, and R. Tkach, "Issues for routing in the optical layer," Communications Magazine, vol. 39, no. 2, pp. 81-87, February 2001. 